\title{
An RSSI-based Scheme for Sybil Attack Detection in Wireless Sensor Networks
}

\author{
Murat Demirbas, Youngwhan Song \\ Department of Computer Science and Engineering Department \\ State University of New York at Buffalo \\ Buffalo, NY 14260-2000 \\ Email: \{demirbas, ywsong\}@ cse.buffalo.edu
}

\begin{abstract}
A sybil node impersonates other nodes by broadcasting messages with multiple node identifiers (ID). In contrast to existing solutions which are based on sharing encryption keys, we present a robust and lightweight solution for sybil attack problem based on received signal strength indicator (RSSI) readings of messages. Our solution is robust since it detects all sybil attack cases with $100 \%$ completeness and less than a few percent false positives. Our solution is lightweight in the sense that alongside the receiver we need the collaboration of one other node (i.e., only one message communication) for our protocol. We show through experiments that even though RSSI is time-varying and unreliable in general and radio transmission is non-isotropic, using ratio of RSSIs from multiple receivers it is feasible to overcome these problems.
\end{abstract}

\section{INTRODUCTION}

The term sybil attack is introduced in [2] to denote an attack where the attacker (sybil node) tries to forge multiple identification in a certain region. Sybil attack is particularly easy to perform in wireless sensor networks (WSN) where the communication medium is broadcast, and same frequency is shared among all nodes. By broadcasting messages with multiple identifications, a sybil node can rig the vote on groupbased decisions and also disrupt network middleware services severely.

Existing solutions for sybil attack prevention are too costly for the resource-poor sensor platforms, such as the popular Berkeley mote platform [6]. Motes (nodes) have very limited computational resources (e.g., 8K RAM, 4Mhz CPU) and are energy constrained; thus, algorithms that impose an excessive communication burden on nodes are not acceptable since they drain the battery power quickly. Solutions [4], [10] that adopt key exchange to vouch identification severely effect the energy consumption due to distribution and piggybacking of randomly generated keys in messages. Moreover, they consume precious memory space as every node is required to store pairwise keys with neighbors.

A received signal strength indicator (RSSI) based solution for sybil attack is desirable as it does not burden the WSN with shared keys or require piggybacking of keys to messages. Ideally, upon receiving a message, the receiver will associate the RSSI of the message with the sender-id included in the message, and later when another message with same RSSI but with different sender-id is received, the receiver would complain of a sybil attack. However, due to the unreliable, time-varying nature of RSSI [8], [15], this straightforward scheme fails. Moreover, since it is very easy to change the transmission power in WSN [3], a sybil node can send messages with different IDs using varying transmission power to trick the receiver. Since RSSI is a function of transmission power, different transmission powers will lead to different RSSI readings.

\section{Contributions of this paper}

In this paper, we report on our implementation of a robust and lightweight solution for detecting sybil attack in WSN using RSSI. Our solution is robust since it detects all sybil attack cases with $100 \%$ completeness and very good accuracy (less than a few percent false positives.) Our solution is lightweight in the sense that alongside the receiver we need the collaboration of one other node (i.e., only one message communication) for our protocol. To the best of our knowledge, this is the first implemented solution for sybil attack detection on the WSN platform.

We show through experiments that even though RSSI is unreliable and time-varying in general and radio transmission is non-isotropic [15], using ratio of RSSIs from multiple receivers it is possible to overcome these problems easily. Use of ratio of RSSIs from multiple receivers was introduced in [14], however, this is the first time that this technique is implemented in practice. We show through experiments that using one receiver there is a lot of variation on RSSI values, however using multiple receivers and ratio of RSSIs the timevariance of RSSI is overcome and the standard deviation is very small. We give confidence intervals for this variance from our experiments at varying distances.

To achieve a lightweight solution, we first point out that we do not need calculation of sender's position. So we relax the computation requirements of [14] by avoiding calculation of fading through distance. Moreover, we show through experiments that even for a 3-D coordinate system, for sybil node detection, two nodes is enough rather than four receiver nodes that is required in the theory [14]. We show that using two receivers $100 \%$ completeness and less than a few percent false positive rate is possible in practice.

Outline: After the preliminaries section, in Section III we present our protocol and investigate the variance in RSSI values and ratio of RSSI values from multiple receivers. In 
Section IV we discuss the experiments with varying number of detector nodes. Finally, we conclude in Section V.

\section{PRELIMINARIES}

In this section, we first define the sybil attack problem, and discuss our implementation platform. We then provide a brief summary of the RSSI-based localization protocol in [14] which we base our work.

\section{A. Problem Statement}

We assume a static network, where all nodes are immobile after initial deployment. We assume an initial set of nodes that are trustworthy (non-sybil). Later, as part of re-populating the network, new nodes are introduced some of which can be sybil. New nodes may be arriving to the network also due to topology-control and sleep-wake up protocols: Previously sleeping nodes might become active later as part of load balancing [5]. Some of these new nodes may be sybil. Note that sybil nodes can vary their transmission power between transmissions to trick other nodes.

- Completeness: If there is a sybil attack in the network, the protocol should detect the sybil attack with probability, greater than $99 \%$.

- Accuracy: The protocol should not identify non-sybil nodes as sybil (as this can ultimately render the WSN useless). In other words, we require the false-positive rate to be less than $10 \%$.

\section{B. Platform}

Hardware: The hardware we use is the Mica2 motes [6], [7] with CC1000 chip [1] using $433 \mathrm{MHz}$ radio frequency and FSK. Mica2 has Atmega128 chip for the processor which is running at $4 \mathrm{MHz}$ clock frequency. Mica2 has $128 \mathrm{~KB}$ of flash memory, 4KB SRAM and 4KB EEPROM. All of our RSSI experiments are done in a large in-door environment.

Software: We implement our protocol on TinyOS version 1.1. [3]. We use the default MAC layer, BMAC [11]. Detailed software structure and codes are at http://www.cse.buffalo.edu/ ywsong/data/ yw_Sybil_SourceCode.zip.

\section{Localization with power}

In contrast to simplistic representations of radio signal as isotropic, and communication range as uniform, [8] and [15] show that broadcast is non-isotropic in WSN. Therefore, using RSSI values directly for sybil attack detection is unreliable. An RSSI-based localization scheme that uses ratio of RSSIs from multiple receivers to overcome to this problem is introduced in [14]. Theorem 5 in [14] argues that if at least four sensors monitor radio signals, then no user can hide its location. Suppose node $i$ receives radio signal from node 0 , then the RSSI is $R_{i}=P_{0} K / d_{i}^{\alpha}$ where $P_{0}$ represents transmitter power, $R_{i}$ is RSSI, $K$ is constant, $d_{i}$ is Euclidean distance, and $\alpha$ is distance-power gradient.

The RSSI ratio of node $i$ to $j$ is

$$
R_{i} / R_{j}=\left(\frac{P_{0} \cdot K}{d_{i}^{\alpha}}\right) /\left(\frac{P_{0} \cdot K}{d_{j}^{\alpha}}\right)=\left(\frac{d_{i}}{d_{j}}\right)^{\alpha}
$$

and the user's location $(x, y)$ can be computed by solving following equation through four receivers, $i, j, k$, and $l$ :

$$
\begin{aligned}
\left(x-x_{i}\right)^{2}+\left(y-y_{i}\right)^{2} & =\left(\frac{R_{i}}{R_{j}}\right)^{\frac{1}{\alpha}}\left(\left(x-x_{j}\right)^{2}+\left(y-y_{j}\right)^{2}\right) \\
& =\left(\frac{R_{i}}{R_{k}}\right)^{\frac{1}{\alpha}}\left(\left(x-x_{k}\right)^{2}+\left(y-y_{k}\right)^{2}\right)(2) \\
& =\left(\frac{R_{i}}{R_{l}}\right)^{\frac{1}{\alpha}}\left(\left(x-x_{l}\right)^{2}+\left(y-y_{l}\right)^{2}\right)
\end{aligned}
$$

, where $x_{i}$ and $y_{i}$ is the location of node $i$. Note that since $P_{0}$ values cancel out in the ratio of RSSIs, this technique is unaffected by the changes to the transmission power $P_{0}$.

\section{RSSI-BASED SYBIL NODE DETECTION}

We first present our RSSI-based sybil attack detection protocol in section III-A, and in section III-B we show that, in contrast to nonuniform nature of individual RSSI values, ratio of RSSI values recorded of multiple receivers exhibit a Gaussian PDF, and, hence, are suitable for detection of sybil nodes.

\section{A. Basic Algorithm}

It is possible to use the localization algorithm in [14] to detect a sybil attack as follows. Upon receiving a message, the four detector nodes compute the location of sender using equation 2 and associate this location with the sender-ID included in the message. Later when another message with different sender-ID is received and the location of the sender is computed to be the same as the previous one, the nodes detect a sybil attack.

However, it is very cumbersome to calculate the location using equation 2 for every node. Indeed, we do not need this calculation for sybil node detection. Since all of $x, y$, and $x_{i}, y_{i}$ location stays the same, it is possible to detect sybil attack by just recording and comparing the ratio of RSSI for the received messages.

Here, we describe our protocol in terms of a scenario. Let four monitoring nodes have ID as $D 1, D 2, D 3$, and $D 4$ respectively and a sybil node forge its ID as $S 1$ and $S 2$ in time. Here is an example topology in Figure 1.

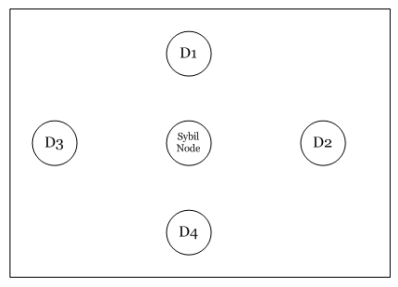

Fig. 1. Example topology

At time $t_{1}$, a sybil node broadcasts a message with its forged ID as $S 1$. Monitoring nodes record the RSSI and the forged ID. Each monitoring node sends a message to $D 1$ containing the received RSSI from $S 1$. Let $R_{i}^{k}$ denote the RSSI value when a message from a sender $k$ is received at $i$. Then, accumulating the messages from the monitors, $D 1$ computes each ratio

$$
\frac{R_{D 1}^{S 1}}{R_{D 2}^{S 1}}, \frac{R_{D 1}^{S 1}}{R_{D 3}^{S 1}}, \text { and } \frac{R_{D 1}^{S 1}}{R_{D 4}^{S 1}}
$$


and stores them locally.

At time $t_{2}$, the sybil node broadcasts a message again with a different ID, $S 2$. The monitoring nodes record the RSSI from $S 2$ and report to $D 1$. $D 1$ computes each ratio as before:

$$
\frac{R_{D 1}^{S 2}}{R_{D 2}^{S 2}}, \frac{R_{D 1}^{S 2}}{R_{D 3}^{S 2}}, \text { and } \frac{R_{D 1}^{S 2}}{R_{D 4}^{S 2}}
$$

Now, $D 1$ can detect the sybil node by comparing the ratio at time $t_{1}$ and $t_{2}$. If the difference between two ratios is very close to zero, $D 1$ concludes that a sybil attack occurred in the region. Since RSSI ratios are same, the location is in fact the same for the alleged multiple IDs. Otherwise, $D 1$ concludes that there is no sybil node. That is, if

$$
\left(\frac{R_{D 1}^{S 1}}{R_{D 2}^{S 1}}=\frac{R_{D 1}^{S 2}}{R_{D 2}^{S 2}}\right),\left(\frac{R_{D 1}^{S 1}}{R_{D 3}^{S 1}}=\frac{R_{D 1}^{S 2}}{R_{D 3}^{S 2}}\right),\left(\frac{R_{D 1}^{S 1}}{R_{D 4}^{S 1}}=\frac{R_{D 1}^{S 2}}{R_{D 4}^{S 2}}\right)
$$

is true, $D 1$ detects a sybil attack.

Later, in section IV-A, we show through experiments that even for a 3-D coordinate system, for sybil node detection, two nodes is enough rather than four receiver nodes that is required in the theory [14].

\section{B. Variance of RSSI}

Ideally, RSSI should stay the same if the locations of the two transceivers are fixed, but even in this case RSSI fluctuates a lot in practice. Here we quantify over this fluctuation by experiments, and we investigate the variance of RSSI and how we can overcome it.

Setup1: We deploy a node to transmit "Hello" messages with constant power $(0 \mathrm{dBm})$. Another node acts as a receiver, captures RSSIs ${ }^{1}$, and transmits them to the PC through RSC232 serial interface ${ }^{2}$. The transmitter sends messages over 2000 times. We set the distance between the transmitter and receiver as $30 \mathrm{~cm}$. We repeat the experiment by changing distance to $1 \mathrm{~m}$.

Result1: Two of the histogram in Figure 2 demonstrates the nonuniform nature of RSSI. The poor correlation of RSSI values makes it unsuitable for detection of sybil attacks.

Setup2: Here, we use two receivers (instead of one) and compare ratio of RSSIs at the two receivers (instead of absolute value of RSSIs) to cope with time varying nature of RSSI. Note that using ratios of RSSIs also takes care of varied transmission power at a sender. In this setup the sender broadcasts messages 2000 times using different (random) transmission power each time. The two receivers record RSSI values and transmit them to the base station which is connected to PC. We repeat experiment twice for distance of $1 \mathrm{~m}$.

For the analysis, the basestation computes the ratio of two RSSI values it received from the two receivers at time $t 1$, and later does the same for RSSIs received at time $t 2$. Then it

\footnotetext{
${ }^{1}$ TinyOS provides RSSI reading for each received message automatically via TOS_Msg- > strength

${ }^{2}$ We use a programming board as MIB510 which is connected to PC through RSC-232 serial interface running at $115200 \mathrm{bps}$
}

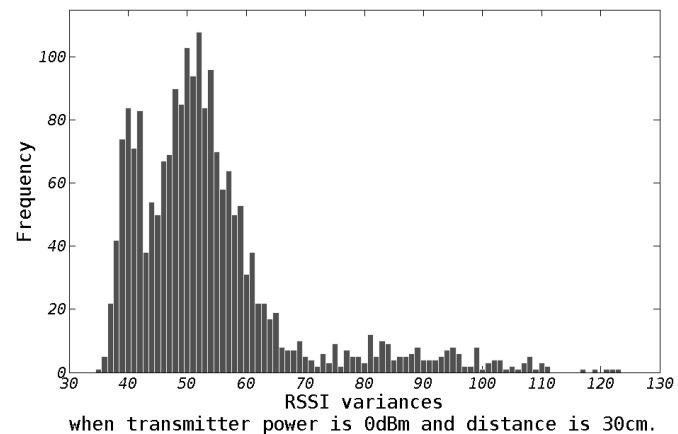

(a) $\rho=51.00, \mu=53.84$, and $\sigma=14.04$ with distance of $30 \mathrm{~cm}$

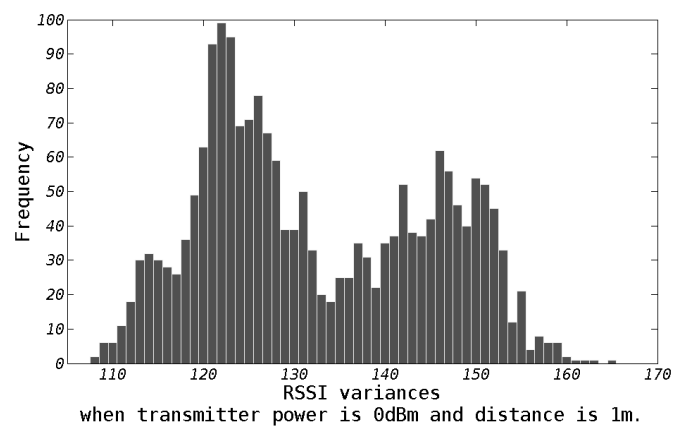

(b) $\rho=129.00, \mu=132.50$, and $\sigma=12.56$ with distance of $1 \mathrm{~m}$

Fig. 2. Variance of RSSI ( $\rho$ stands for median value, $\mu$ for mean, and $\sigma$ for standard deviation)

calculates the difference of two ratios and logs this value. The calculation is repeated throughout the experiment.

Result2: The histogram in Figure 3 shows a uniform distribution of values. The difference of RSSI ratio of 0 dominates over the other values. The values -0.2 and 0.2 occurred only once out of 2000 times $(0.05 \%)$ in Figure 3(a), and similarly those of -0.35 and 0.425 in Figure 3(b). Note that the histogram follows a Gaussian Probability Distribution Function (PDF) with a standard deviation of 0.066 and 0.106 respectively.

If $S 1$ and $S 2$ are different but their location is same, we can infer the sybil attack by noticing that the difference of RSSI ratios for both cases are within a threshold.

$$
\begin{array}{r}
\left(\frac{R_{D 1}^{S 1}}{R_{D 2}^{S 1}}-\frac{R_{D 1}^{S 2}}{R_{D 2}^{S 2}}\right)<\sigma,\left(\frac{R_{D 1}^{S 1}}{R_{D 3}^{S 1}}-\frac{R_{D 1}^{S 2}}{R_{D 2}^{S 2}}\right)<\sigma \\
, \text { and }\left(\frac{R_{D 1}^{S 1}}{R_{D 4}^{S 1}}-\frac{R_{D 1}^{S 2}}{R_{D 4}^{S 2}}\right)<\sigma
\end{array}
$$

The standard deviation in Gaussian PDF covers around $70 \%$ of values, hence setting the threshold to $\sigma$ means that sybil node is detected with $70 \%$ probability. To cover more than $99.999999 \%$, we set the threshold to be $5 \sigma$, more specifically 0.5 in our sybil attack detection experiments.

Setup3: In order to evaluate the effect of distance on $\sigma$, here we repeat the experiment in Setup2 with respect to increasing distances between the transmitter and the receivers. 


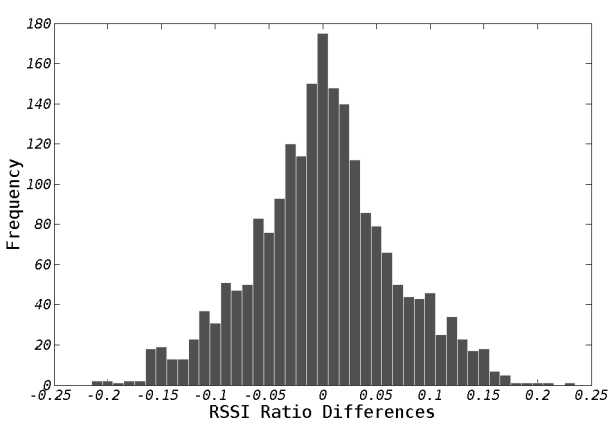

(a) $\rho=0.000, \mu=0.000$, and $\sigma=0.066$

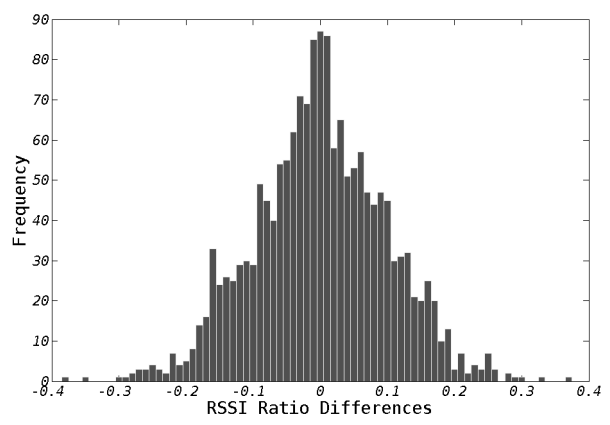

(b) $\rho=0.000, \mu=0.000$, and $\sigma=0.100$

Fig. 3. Variance of difference of RSSI ratio

We performed 100 transmissions at each distance, and vary the distance between $1 \mathrm{~m}$ to $10 \mathrm{~m}$.

Result3: The result is shown in Figure 4. We see that $\sigma$ does not stray away from 0.1 . Therefore, we conclude it is safe to set $\sigma$ as 0.1 and threshold to 0.5 for detection of sybil node attacks.

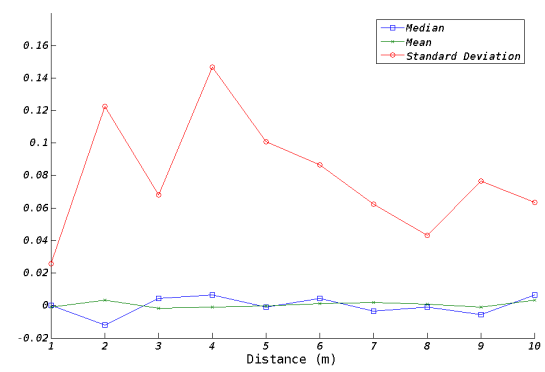

Fig. 4. Various Median, Mean and Standard deviation by distance

Note that this experiment is performed in an in-door environment. According to [13], usually gray area (nondeterministic communication range) starts from 20 meter in in-door environment. All of our experiments and readings fall within the in-band communication range and are not subject to the gray area problems.

\section{EXPERIMENTS}

Here we test our RSSI based sybil attack detection protocol under different setups. First, in Section IV-A we use four receivers for detection. In Section IV-B, we use only two receivers and show that the protocol achieves a similar performance to the four receiver case.

\section{A. Sybil experiments with four detectors}

We perform two experiments, the first for evaluating completeness, second for accuracy. The first experiment uses the topology in Figure 5(a), and the second in Figure 5(b).

Setup4: Figure 5(a) shows a sybil node and four receiver nodes in the network. When a sybil node sends a message, each of the four monitors records the RSSI value and ID associated with the message, and $D 2, D 3$, and $D 4$ send their readings to $D 1$. When the sybil performs another broadcast with different ID and different transmission power, the monitors record the readings for this message, and the information is again accumulated at $D 1$. Next $D 1$ detects whether there is a sybil attack in the network using Equation 6. We set the threshold to be $5 \sigma$, which is 0.5 according to Section III-B. To avoid message collisions during the experiment, we regulate the message transmission times using timers at each node. In our experiment, the sybil node broadcasts every 30 seconds, and each receiver transmit to D1 with 3 second intervals after the sybil node's detection.

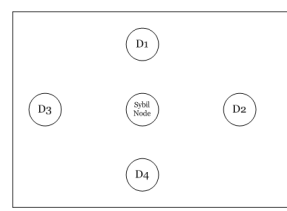

(a) Topology in case of (b) Topology in case of four monitoring nodes and four monitoring nodes and one sybil node no sybil node. Gx represents good nodes, Dx, detectors
Fig. 5. Sybil attack experiment with four detectors

Result4: We repeated the above experiment 100 times, before each instance we changed the deployment location of receiving nodes and the sybil node. Using 30 second intervals for sybil node transmission meant that we changed the topology once every minute. We saw that node D1 always detected the sybil attack in all instances.

Setup5: To test for accuracy (absence of false-positives), we changed the setup to the topology in Figure 5(b). Here, we eliminated the sybil node in the network, and deployed two good nodes which broadcasts only their own IDs. The good nodes used varying transmission powers [9]. Due to energyefficiency purposes some protocols require nodes to transmit with varying transmission power, also as the battery power decreases and environmental factors change transmission power inevitably changes. Note that, since the receivers use ratio of RSSI values, the varying transmission powers have no effect in correct evaluation of good nodes versus sybil nodes. We changed the location of good nodes for each run to force a false-positive at the detectors.

Result5: In none of our 100 tries node D1 complained about a sybil attack. Even when the two good nodes are located 
within centimeters of each other, D1 was able to tell that there was no sybil node in the network. Therefore, we conclude that RSSI-based scheme for sybil attack detection using four detectors satisfies our accuracy requirements.

\section{B. Sybil experiments with two detectors}

We again perform two experiments, the first for evaluating completeness, and second for accuracy. The first experiment uses the topology in Figure 6(a), and the second in Figure 6(b).

Setup6: Here we have two receivers and a sybil node. Otherwise, the experiment is performed as in Setup 4. We repeated the experiment 100 times changing the deployment location of nodes in between iterations. Since there are only two receivers, we used only one comparison in Equation (6).
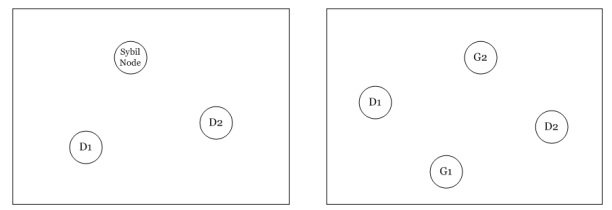

(a) Topology in case of (b) Topology in case of two monitoring nodes and two monitoring nodes and a sybil node no sybil node. Gx represents good nodes, Dx, detectors

Fig. 6. Sybil attack experiment with two detectors

Result6: Similar to Result4, node D1 always detected a sybil attack.

Setup7: In order to see that the detectors can distinguish whether there is a sybil node or not, we use the setup in Figure 6(b). In this setup, there are two monitors and two good nodes. Each good node has distinct ID and uses varying transmission power. The experiment was repeated 100 times changing location of good nodes to force a false-positive at the detectors.

Result7: 3 cases out of 100 times, node D1 detected a sybil attack inaccurately. That is, when using only two receivers, upto 5\% false-positives may be possible.

Remark: These false-positive experiments are best effort in that they do not provide an absolute scale for false-positives for any environment or distance. Rather, they help us compare the false-positive rates when using varying number of detectors, as we try to perform the false-positive experiments similarly for each case. (End of remark)

Note that in the two receiver case, only one transmission is enough for sybil node detection, that of node $D 2$ to $D 1$. Hence, the overhead is very low in this case, but the tradeoff is the increased false-positive rate. However, for sybil attack problem completeness is more critical than the accuracy: Not detecting a sybil node has severe implications for security, whereas falsely detecting upto $5 \%$ of nodes as sybil has only implications in reducing the system performance. Based on this observation, we suggest that RSSI-based scheme for sybil attack detection using two detectors is more suitable than the four node version for practical deployments.

\section{CONCLUDING REMARKS}

We presented an RSSI-based solution for the sybil attack problem in WSN. We showed that even though RSSI is timevarying and unreliable in general and radio transmission is non-isotropic, using ratio of RSSIs from multiple receivers it is feasible to overcome these problems. Our protocol is lightweight—alongside the receiver we need the collaboration of one other node - and robust-we achieve detection with $100 \%$ completeness and less than a few percent false positives.

In future work we will try to answer how we can extend our protocol to tolerate existing sybil nodes in the network. We will test our protocol in a large-scale WSN testbed, Kansei [12], in preparation for using our solution in real deployments.

\section{REFERENCES}

[1] Chipcon. Cc1000 radio datasheet. www.chipcon.com/files/ CC1000_Data_Sheet_2_3.pdf, 2003.

[2] J. R. Douceur. The sybil attack. In IPTPS, pages 251-260, 2002.

[3] D. Gay, P. Levis, R. von Behren, M. Welsh, E. Brewer, and D. Culler The nesc language: A holistic approach to networked embedded systems. In PLDI, pages 1-11, 2003.

[4] P. Golle, D. Greene, and J. Staddon. Detecting and correcting malicious data in vanets. In VANET, pages 29-37, 2004.

[5] W. R. Heinzelman, A. Chandrakasan, and H. Balakrishnan. Energyefficient communication protocol for wireless microsensor networks. In HICSS, page 8020, 2000.

[6] J. Hill and D. Culler. Mica: A wireless platform for deeply embedded networks. volume 22(6), pages 12-24, Nov/Dec 2002.

[7] J. Hill, R. Szewczyk, A. Woo, S. Hollar, D. Culler, and K. Pister. System architecture directions for network sensors. ASPLOS, pages 93-104, 2000.

[8] D. Kotz, C. Newport, R. S. Gray, J. Liu, Y. Yuan, and C. Elliott. Experimental evaluation of wireless simulation assumptions. In MSWiM, pages 78-82, 2004.

[9] L. Li, J. Halpern, P. Bahl, Y.-M. Wang, and R. Wattenhofer. A cone-based distributed topology-control algorithm for wireless multihop networks. IEEE/ACM Trans. Netw., 13(1):147-159, 2005.

[10] J. Newsome, E. Shi, D. Song, and A. Perrig. The sybil attack in sensor networks: analysis \& defenses. In IPSN, pages 259-268, 2004.

[11] J. Polastre, J. Hill, and D. Culler. Versatile low power media access for wireless sensor networks. In SenSys, pages 95-107, 2004.

[12] OSU NEST ExScal Team. Kansei: Sensor testbed for at-scale experiments. http://www.cse.ohio-state.edu/kansei.

[13] J. Zhao and R. Govindan. Understanding packet delivery performance in dense wireless sensor networks. In SenSys, pages 1-13, 2003.

[14] S. Zhong, L. Li, Y. G. Liu, and Y. R. Yang. Privacy-preserving locationbased services for mobile users in wireless networks. Technical Report YALEU/DCS/TR-1297, Yale Computer Science, July 2004.

[15] G. Zhou, T. He, S. Krishnamurthy, and J. A. Stankovic. Impact of radio irregularity on wireless sensor networks. In MobiSys, pages 125-138, 2004. 